\title{
A NOTE ON HYPERGEODESICS AND CANONICAL LINES
}

\section{L. MACQUEEN}

In this note we introduce two families of hypergeodesics on a nonruled surface in ordinary projective space. Consideration of the properties of these hypergeodesics leads to certain geometrical constructions which yield canonical lines of the first kind from a given canonical line of the second kind.

We shall assume that the differential equations of a non-ruled surface $S$ are written in the Fubini canonical form ${ }^{1}$

(1) $x_{u u}=p x+\theta_{u} x_{u}+\beta x_{v}, \quad x_{v v}=q x+\gamma x_{u}+\theta_{v} x_{v} \quad(\theta=\log \beta \gamma)$.

We select an ordinary point $P_{x}$ of the surface $S$ as one vertex of the usual local tetrahedron of reference. When a curve $C_{\lambda}$ through the point $P_{x}$ is regarded as being imbedded in the one-parameter family of curves represented on $S$ by the equation

$$
d v-\lambda(u, v) d u=0,
$$

the osculating plane at the point $P_{x}$ of the curve $C_{\lambda}$ has the local equation

$$
2 \lambda\left(\lambda x_{2}-x_{3}\right)+\left(\lambda^{\prime}+\beta-\theta_{u} \lambda+\theta_{v} \lambda^{2}-\gamma \lambda^{3}\right) x_{4}=0,
$$

in which we have placed $\lambda^{\prime}=\lambda_{u}+\lambda \lambda_{v}$.

It will be recalled that two lines $l_{1}(a, b), l_{2}(a, b)$ are reciprocal lines ${ }^{2}$ at a point $P_{x}$ of a surface if the line $l_{1}(a, b)$ joins the point $P_{x}$ and the point $y$ defined by placing

$$
y=-a x_{u}-b x_{v}+x_{u v}
$$

and the line $l_{2}(a, b)$ joins the points $\rho, \sigma$ defined by

$$
\rho=x_{u}-b x, \quad \sigma=x_{v}-a x,
$$

where $a, b$ are functions of $u, v$. As the point $P_{x}$ varies over the surface $S$, the lines $l_{1}(a, b), l_{2}(a, b)$ generate two reciprocal congruences $\Gamma_{1}, \Gamma_{2}$, respectively.

The two reciprocal lines $l_{1}(a, b), l_{2}(a, b)$ are canonical lines $l_{1}(k)$, $l_{2}(k)$ of the first and second kind respectively in case

$$
a=-k \psi, \quad b=-k \phi,
$$

Received by the editors February 2, 1945.

${ }^{1}$ E. P. Lane, Projective differential geometry of curves and surfaces, Chicago, 1932. p. 69.

2 E. P. Lane, loc. cit., pp. 82-85. 
where $k$ is a constant and $\phi, \psi$ are defined by

$$
\phi=\left(\log \beta \gamma^{2}\right)_{u}, \quad \psi=\left(\log \beta^{2} \gamma\right)_{v} .
$$

Canonical lines of the first kind lie in the canonical plane whose local equation is

$$
\phi x_{2}-\psi x_{3}=0 .
$$

The equation of the plane $\pi(a, b)$ which is the harmonic conjugate of the tangent plane of the surface at the point $P_{x}$ with respect to the two focal planes of a general line $l_{2}(a, b)$ of the congruence $\Gamma_{2}$ is given by

$$
x_{1}+b x_{2}+a x_{3}+\left[2^{-1}\left(a_{u}+b_{v}\right)+a b\right] x_{4}=0 .
$$

For a general canonical line $l_{2}(k)$, the local coordinates $\xi_{1}, \cdots, \xi_{4}$ of the plane $\pi(k)$ are given by

(6) $\quad \xi_{1}=1, \quad \xi_{2}=-k \phi, \quad \xi_{3}=-k \psi, \quad \xi_{4}=-3 k \theta_{u v} / 2+k^{2} \phi \psi$.

The equations, in plane coordinates, of the two asymptotic osculating quadrics $Q_{u}, Q_{v}$ of a curve $C_{\lambda}$ are respectively

$$
\begin{array}{r}
2 \lambda^{3}\left(\xi_{2} \xi_{3}-\xi_{1} \xi_{4}\right)-2 \beta \xi_{1}\left(\beta \xi_{1}-\lambda \xi_{2}+\lambda^{2} \xi_{3}\right)-C \xi_{1}^{2}=0, \\
2\left(\xi_{2} \xi_{3}-\xi_{1} \xi_{4}\right)-2 \gamma \lambda \xi_{1}\left(\gamma \lambda \xi_{1}^{2}+\xi_{2}-\lambda \xi_{3}\right)-D \xi_{1}^{2}=0,
\end{array}
$$

where we have placed

$$
\begin{aligned}
& C=\beta\left[\lambda^{\prime}-\beta+\left(\phi-\theta_{u}\right) \lambda-\left(2 \psi-\theta_{v}\right) \lambda^{2}\right]-\left(\beta \gamma+\theta_{u v}\right) \lambda^{3}, \\
& D=\gamma\left[-\lambda^{\prime}-\gamma \lambda^{3}+\left(\psi-\theta_{v}\right) \lambda^{2}-\left(2 \phi-\theta_{u}\right) \lambda\right]-\left(\beta \gamma+\theta_{u v}\right) .
\end{aligned}
$$

Let us now suppose that, at each point $P_{x}$ of the curve $C_{\lambda}$, the asymptotic osculating quadric $Q_{u}$ of $C_{\lambda}$ is tangent to the plane $\pi(k)$. Then we find that the function $\lambda$ satisfies the differential equation

$$
\lambda^{\prime}=A_{1}+B_{1} \lambda+C_{1} \lambda^{2}+D_{1} \lambda^{3}
$$

where the coefficients $A_{1}, B_{1}, C_{1}, D_{1}$ are given by

$$
\begin{array}{ll}
A_{1}=-\beta, & B_{1}=\theta_{u}-(1+2 k) \phi, \\
C_{1}=2(1+k) \psi-\theta_{v}, & D_{1}=(1 / \beta)\left[\beta \gamma+(1+3 k) \theta_{u v}\right],
\end{array}
$$

in which, for the present, we assume $k \neq-1 / 3$, so that the canonical line $l_{2}(k)$ is not the second axis $a_{2}$ of Cech.

Similarly, the asymptotic osculating quadric $Q_{v}$ of $C_{\lambda}$ is tangent to the plane $\pi(k)$ if, and only if,

$$
\lambda^{\prime}=A_{2}+B_{2} \lambda+C_{2} \lambda^{2}+D_{2}^{\prime} \lambda^{3},
$$


where the coefficients $A_{2}, B_{2}, C_{2}, D_{2}$ are given by

$$
\begin{array}{ll}
A_{2}=-(1 / \gamma)\left[\beta \gamma+(1+3 k) \theta_{u v}\right], & B_{2}=\theta_{u}-2(1+k) \phi, \\
C_{2}=(1+2 k) \psi-\theta_{v}, & D_{2}=\gamma,
\end{array}
$$

in which $k \neq-1 / 3$. Thus we reach the following conclusion:

At each point $P_{x}$ of a curve $C_{\lambda}$, each of the asymptotic osculating quadrics $Q_{u}$ and $Q_{v}$ of $C_{\lambda}$ is tangent to the plane $\pi(k)$ which is the harmonic conjugate of the tangent plane of the surface at the point $P_{x}$ with respect to the two focal planes of any canonical line $l_{2}(k)$, except the sec-

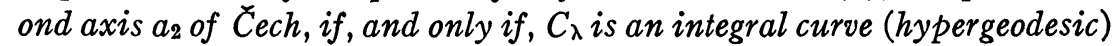
of the respective differential equations (8), (10).

By means of equation (3), together with equation (8), we find that the osculating plane at a point $P_{x}$ of a hypergeodesic of the family (8) has the local equation

$$
\begin{aligned}
& 2 \beta\left(\lambda x_{2}-x_{3}\right) \\
& \quad+\left[-(1+2 k) \beta \phi+2(1+k) \beta \psi \lambda+(1+3 k) \theta_{u v} \lambda^{2}\right] x_{4}=0 .
\end{aligned}
$$

The envelope of the osculating planes at the point $P_{x}$ of all the hypergeodesics of the family (8) is found from equation (12) to be the nondegenerate quadric cone whose equation is

$$
\beta\left[x_{2}+(1+k) \psi x_{4}\right]^{2}+(1+3 k) \theta_{u v}\left[2 x_{3}+(1+2 k) \phi x_{4}\right] x_{4}=0 .
$$

Similarly, the envelope of the osculating planes at the point $P_{x}$ of the hypergeodesics (10) is the quadric cone

$$
\gamma\left[x_{3}+(1+k) \phi x_{4}\right]^{2}+(1+3 k) \theta_{u v}\left[2 x_{2}+(1+2 k) \psi x_{4}\right] x_{4}=0 .
$$

The vertex of each of the cones (13), (14) is, of course, the point $P_{x}$. Furthermore, the cone (13) is tangent to the tangent plane of the surface at the point $P_{x}$ along the asymptotic $v$-tangent at $P_{x}$, while the cone (14) has the asymptotic $u$-tangent of $S$ at $P_{x}$ for its line of contact with the tangent plane. The polar plane of any point on the $u$-tangent with respect to the cone (13) intersects this cone, besides in the $v$-tangent at $P_{x}$, also in a generator which is the line $l_{1}(a, b)$ with $a$ and $b$ defined by the formulas

$$
a=(1+k) \psi, \quad b=2^{-1}(1+2 k) \phi .
$$

We may also regard this line as being determined by the planes whose equations are

$$
x_{2}+(1+k) \psi x_{4}=0, \quad x_{3}+2^{-1}(1+2 k) \phi x_{4}=0 .
$$

Similarly, the polar plane of any point on the $v$-tangent with re- 
spect to the cone (14) intersects this cone in the $u$-tangent at $P_{x}$ and in the line $l_{1}(a, b)$ for which

$$
a=2^{-1}(1+2 k) \psi, \quad b=(1+k) \phi .
$$

This line may also be regarded as determined by the planes

$$
x_{2}+2^{-1}(1+2 k) \psi x_{4}=0, \quad x_{3}+(1+k) \phi x_{4}=0 .
$$

The locus of the line (16) for all canonical lines $l_{2}(k)$ is found by eliminating $k$ from equations (16) to be the plane

$$
\phi x_{2}-\psi x_{3}+2^{-1} \phi \psi x_{4}=0 \text {. }
$$

Similarly, the locus of the line (18) is the plane

$$
\phi x_{2}-\psi x_{3}-2^{-1} \phi \psi x_{4}=0 .
$$

Thus we find that the tangent plane of the surface at the point $P_{x}$ and the canonical plane (4) separate the planes defined by equations (19), (20) harmonically.

We now describe simple geometrical constructions which yield canonical lines of the first kind from a given canonical line $l_{2}(k)$ of the second kind, except the second axis $a_{2}$ of Cech. In the first place, the plane determined by the two lines (16), (18) has the equation

$$
\phi x_{2}+\psi x_{3}+2^{-1}(3+4 k) \phi \psi x_{4}=0
$$

and is found to intersect the canonical plane (4) in the canonical line $l_{1}\left(k_{1}\right)$ for which

$$
k_{1}=-4^{-1}(3+4 k)
$$

The polar plane of any point on the $u$-tangent at $P_{x}$ with respect to the cone (13) intersects the polar plane of any point on the $v$-tangent at $P_{x}$ with respect to the cone (14) in the canonical line $l_{1}\left(k_{2}\right)$ for which

$$
k_{2}=-(1+k) \text {. }
$$

Furthermore, the plane which is tangent to the cone (13) along the line (16) intersects the plane which is tangent to the cone (14) along the line $(18)$ in the canonical line $l_{1}\left(k_{3}\right)$ for which

$$
k_{3}=-2^{-1}(1+2 k) \text {. }
$$

We remark that if the line $l_{2}(k)$ is the second edge $e_{2}$ of Green, for which $k=-1 / 4$, then the three canonical lines $l_{1}\left(k_{1}\right), l_{1}\left(k_{2}\right)$, and $l_{1}\left(k_{3}\right)$ obtained by the preceding constructions are respectively the first di- 
rectrix $d_{1}$ of Wilczynski, the first canonical line for which $k=-3 / 4$, and the first edge $e_{1}$ of Green.

It may be seen from the formulas (22), (23), and (24) that a given canonical line $l_{2}(k)$ will yield in turn each of the canonical lines $l_{1}\left(k_{1}\right)$, $l_{1}\left(k_{2}\right)$, and $l_{1}\left(k_{3}\right)$ for its reciprocal if, and only if, the given canonical line $l_{2}(k)$ is the second canonical line for which $k=-3 / 8$, the second directrix $d_{2}$ of Wilczynski, and the second edge $e_{2}$ of Green.

Finally, if the given canonical line $l_{2}(k)$ is the second axis $a_{2}$ of Cech for which $k=-1 / 3$, the asymptotic osculating quadric $Q_{u}$ of $C_{\lambda}$ is tangent to the plane $\pi(-1 / 3)$ if, and only if,

$$
\lambda^{\prime}=-\beta+\left(\theta_{u}-\phi / 3\right) \lambda+\left(4 \psi / 3-\theta_{v}\right) \lambda^{2}+\gamma \lambda^{3},
$$

so that the curve $C_{\lambda}$ is a union curve of the congruence $\Gamma_{1}$ of lines $l_{1}(a, b)$ for which

$$
a=2 \psi / 3, \quad b=\phi / 6 .
$$

Similarly, the asymptotic osculating quadric $Q_{v}$ of $C_{\lambda}$ is tangent to the plane $\pi(-1 / 3)$ if, and only if,

$$
\lambda^{\prime}=-\beta+\left(\theta_{u}-4 \phi / 3\right) \lambda+\left(\psi / 3-\theta_{v}\right) \lambda^{2}+\gamma \lambda^{3},
$$

in which case $C_{\lambda}$ is a union curve of the congruence $\Gamma_{1}$ of lines $l_{1}(a, b)$ for which

$$
a=\psi / 6, \quad b=2 \phi / 3 .
$$

The plane determined by the two lines thus defined at the point $P_{x}$ intersects the canonical plane in the canonical line $l_{1}(k)$ for which $k=-5 / 12$, namely, the first axis of Bompiani.

We conclude with the statement that if at the point $P_{x}$ both of the asymptotic osculating quadrics $Q_{u}$ and $Q_{v}$ of $C_{\lambda}$ are tangent to the plane $\pi(-1 / 3)$, then $\phi+\psi \lambda=0$, so that the curve $C_{\lambda}$ is tangent at $P_{x}$ to the second canonical tangent $t_{2}$.

SOUthwestern College 